\title{
Biomarkers of Parkinson's disease: recent insights, current challenges, and future prospects
}

\author{
This article was published in the following Dove Press journal: \\ Journal of Parkinsonism and Restless Legs Syndrome \\ 22 February 2016 \\ Number of times this article has been viewed
}

\author{
Marina Picillo' \\ Marcello Moccia ${ }^{2}$ \\ Emanuele Spina ${ }^{2}$ \\ Paolo Barone' \\ Maria Teresa Pellecchia' \\ 'Department of Medicine and Surgery, \\ Center for Neurodegenerative \\ Diseases (CEMAND), Neuroscience \\ Section, University of Salerno, \\ Salerno, Italy; ${ }^{2}$ Department of \\ Neuroscience, Reproductive and \\ Odontostomatologic Sciences, \\ Federico II University, Naples, Italy
}

\begin{abstract}
A biomarker represents a tool possibly helping physicians in predicting onset, diagnosis, and progression of a disease as well as evaluating the response to disease-modifying treatments. Currently, there is no biomarker fulfilling all such ideal criteria for Parkinson's disease (PD). In this article, we have critically reviewed the literature searching for the most reliable and reproducible clinical, biochemical, and imaging biomarkers for prodromal phase, diagnosis, and progression of PD. Different comprehensive batteries of biomarkers have been proposed as a sensitive approach to predict the onset of PD during the prodromal phase. There is a discussion about the redefinition of the clinical diagnosis of PD, including clinical biomarkers as non-motor symptoms; however, on the other hand, we have also observed that imaging biomarkers support the differential diagnosis from other causes of parkinsonism. Various clinical (eg, freezing of gait or cognitive impairment), biochemical (eg, epidermal growth factor, insulin-like growth factor 1, uric acid, etc), and imaging (eg, functional magnetic resonance imaging, voxel-based morphometry, etc) biomarkers may help envisaging disease progression of PD. To conclude, given the lack of a single biomarker that could track the entire course of the disease, our challenge is to find the best combinations of biomarkers for the different stages of the disease.
\end{abstract}

Keywords: biomarkers, Parkinson's disease, progression, motor, imaging, staging, non motor

\section{Introduction}

The National Institutes of Health defines a biomarker as "a characteristic that is objectively measured and evaluated as an indicator of normal biological processes, pathogenic processes or pharmacological responses to a therapeutic intervention."1 In the field of neurodegenerative diseases, a biomarker represents a tool possibly helping physicians in predicting onset, diagnosis, and progression of a disease as well as evaluating the response to disease-modifying treatments (DMT).

Parkinson's disease (PD) is the second most common neurodegenerative disease after Alzheimer's disease, affecting up to 1 million Americans over age 65 and up to 10 million individuals worldwide. ${ }^{2}$ DMT as well as a cure for PD have yet to come. Current research is focusing on finding reliable biomarkers for PD which, ideally, should be sensitive, reproducible, inexpensive, noninvasive, and thoroughly validated. How can biomarkers really help us to tackle PD? The aim of this review is to find an answer to such a question by summarizing the current knowledge on clinical, imaging, and biochemical biomarkers for prodromal phase, diagnosis, and disease progression of PD (Tables 1-3).
Correspondence: Maria Teresa Pellecchia Department of Medicine and Surgery, Center for Neurodegenerative Diseases (CEMAND), Neuroscience Section, University of Salerno,Via Allende 8408I, Salerno, Italy

Tel/fax +39089672328

Emailmpellecchia@unisa.it
Journal of Parkinsonism and Restless Legs Syndrome 2016:6 I-13

(c) (1) (\$) $\odot 2016$ Picillo et al. This work is published and licensed by Dove Medical Press Limited. The full terms of this license are available at https://www.dovepress.com/terms. cc. you hereby accept the Terms. Non-commercial uses of the work are permitted without any further permission from Dove Medical Press Limited, provided the work is properly attributed. For permission for commercial use of this work, please see paragraphs 4.2 and 5 of our Terms (https://www.dovepress.com/terms.php). 
Table I Biomarkers for PD prodromal phase

\begin{tabular}{|c|c|c|}
\hline Biomarker & Comments & References \\
\hline \multicolumn{3}{|l|}{ Clinical biomarkers } \\
\hline \multirow[t]{22}{*}{ Non-motor symptoms (NMS) } & Family history for either PD or tremor & 4 \\
\hline & Lack of smoking history & 4,5 \\
\hline & More frequent NMS 2 years before diagnosis: & 3 \\
\hline & anhedonia & 3 \\
\hline & apathy & 3 \\
\hline & memory complaints & 3 \\
\hline & inattention & 3 \\
\hline & More frequent NMS 2-10 years before diagnosis: & 3 \\
\hline & smell loss & $3,10-12$ \\
\hline & mood disturbances & 3 \\
\hline & excessive sweating & 3 \\
\hline & fatigue & 3 \\
\hline & pain & 3 \\
\hline & other factors: tremor, balance impairments, constipation, hypotension, erectile & $6-9$ \\
\hline & dysfunction, urinary dysfunction, dizziness, fatigue, depression, and anxiety. & \\
\hline & More frequent NMS more than 10 years before diagnosis: & 3 \\
\hline & constipation & 3,9 \\
\hline & dream-enacting behavior & $3,10-12$ \\
\hline & frequent nightmares & $3,10-12$ \\
\hline & excessive daytime sleepiness & 3 \\
\hline & postprandial fullness & 3 \\
\hline & other factor: tremor & 9 \\
\hline \multicolumn{3}{|l|}{ Biochemical biomarkers } \\
\hline Metabolic factors & Cholesterol may be either a protective or a risk factor for PD & 14,15 \\
\hline \multirow[t]{2}{*}{ Neurotrophic factors } & High IGF-I & 16,17 \\
\hline & Low vitamin $\mathrm{D}$ & 18 \\
\hline Oxidative stress & Protective role of higher uric acid levels and of gout in the development of PD & $19-23$ \\
\hline \multicolumn{3}{|l|}{ Imaging biomarkers } \\
\hline Nuclear imaging & $\begin{array}{l}\text { DaT imaging (DaTscan) is used to detect nigrostriatal degeneration; if combined with } \\
\text { multiple factors (hyposmia, male sex, and constipation), the probability of detecting } \\
\text { subjects with a nigrostriatal deficit increases up to } 40 \% \text {. }\end{array}$ & $24-26$ \\
\hline \multirow[t]{3}{*}{ Transcranial sonography (TCS) } & $\begin{array}{l}\text { Substantia nigra hyperechogenicity has been proposed as a prodromal biomarker } \\
\text { of PD at both 3- and 5-year follow-up. }\end{array}$ & 27,28 \\
\hline & Sensitivity and specificity of PD conversion can be significantly increased when & $29-31$ \\
\hline & TCS is associated with nuclear imaging or applied in LRRK2 mutation carriers. & \\
\hline Magnetic resonance imaging (MRI) & Functional MRI shows reorganization of corticostriatal circuits in $L R R K 2$ mutation carriers & 32 \\
\hline
\end{tabular}

Note: DaTscan signifies dopamine transporter imaging technique.

Abbreviations: PD, Parkinson's disease; IGF, insulin-like growth factor.

\section{Methods}

We searched the published data in English language from inception to November 2015 on PubMed. Our keywords included "Parkinson's disease", "biomarkers", "imaging", "clinical", "serum", "plasma", and "cerebrospinal fluid or CSF". Totally, we have retrieved 1,601 papers. Out of these, however, we considered only 141 papers for this review after excluding preclinical studies, reviews, duplicated data, and papers not specifically focused on PD.

\section{Prodromal phase Clinical biomarkers}

Several retrospective studies have examined the frequency and timeline of clinical biomarkers prior to the onset of overt PD motor symptoms. In the ONSET PD (onset of nonmotor symptoms in PD) study, the occurrence of non-motor symptoms (NMS) prior to diagnosis was surveyed in $109 \mathrm{PD}$ patients with an estimation of the time span between onset of NMS and motor symptoms (within 2 years; more than 2 but less than 10 years; and more than 10 years). The most common NMS reported to occur more frequently during the 2-year premotor period were anhedonia, apathy, memory complaints, and inattention. NMS occurring more frequently between 2 and 10 years before motor onset were smell loss and mood disturbances, taste loss, excessive sweating, fatigue, and pain. NMS occurring usually more than 10 years before motor onset were constipation, dream-enacting behavior and frequent nightmares (both suggestive of rapid eye movement [REM] 
Table 2 Biomarkers for PD diagnosis

\begin{tabular}{|c|c|c|}
\hline Biomarkers & Comments & References \\
\hline \multicolumn{3}{|l|}{ Clinical biomarkers } \\
\hline \multirow[t]{4}{*}{ Non-motor symptoms (NMS) } & Frequent NMS at diagnosis: psychiatric disturbances, fatigue, attention/memory problems & 34 \\
\hline & Sleep symptoms (RBD and insomnia) associated with worse cognitive performances & 35 \\
\hline & Taste/smelling and difficulties in sexual activities more prevalent in men & 36 \\
\hline & Role of "red flags" in differential diagnosis with multiple system atrophy (MSA) & 41 \\
\hline \multicolumn{3}{|l|}{ Biochemical biomarkers } \\
\hline Metabolic factors & Increased homocysteine and its metabolites (ie, methylmalonate or cystatin C) & $42-44$ \\
\hline \multirow[t]{4}{*}{ Neurotrophic factors } & Low BDNF & 46 \\
\hline & High IGF-I & $16,48,49$ \\
\hline & Low vitamin $\mathrm{D}$ & 50,51 \\
\hline & GH response to arginine test may be helpful in the differential diagnosis with MSA & 47 \\
\hline Neuroinflammation & PDGFB and prolactin are possible biomarkers for PD & 52,53 \\
\hline \multirow[t]{2}{*}{ Oxidative stress } & Bilirubin and ceruloplasmin have been variably associated with PD & $54-57$ \\
\hline & Lower levels of uric acid in PD patients compared to healthy controls & 58,59 \\
\hline Iron and other metals & Iron and manganese have been variably associated with PD & 62,63 \\
\hline$\alpha$-Synuclein & $\begin{array}{l}\text { It is still debated if plasmatic levels of } \alpha \text {-synuclein are similar or higher in PD, as compared } \\
\text { to healthy controls; different methods to detect } \alpha \text {-synuclein in plasma possibly account for } \\
\text { such divergent results. }\end{array}$ & $64-67$ \\
\hline \multirow[t]{5}{*}{ CSF biomarkers } & Altered $A \beta$ peptides & $68-70$ \\
\hline & Decreased $\alpha$-synuclein & 71,72 \\
\hline & Higher tau protein & 74 \\
\hline & Higher DJ-I & 75 \\
\hline & Metabolomic studies & 79,80 \\
\hline \multicolumn{3}{|l|}{ Imaging biomarkers } \\
\hline \multirow[t]{3}{*}{ Nuclear imaging } & $\begin{array}{l}\text { Evaluation of the presynaptic dopaminergic function by means of either 6-[I8F] fluoro-L-dopa } \\
\text { or VMAT } 2 \text { or DAT availability measurement can be helpful in differentiating degenerative } \\
\text { parkinsonism from essential tremor and secondary parkinsonism, but it is not helpful in the } \\
\text { differential diagnosis with atypical parkinsonism. }\end{array}$ & 81,82 \\
\hline & $\begin{array}{l}\text { Cardiac }\left[{ }^{[23} \mid\right] \text { metaiodobenzylguanidine (MIBG) scintigraphy may also be useful for the } \\
\text { differential diagnosis with MSA. }\end{array}$ & 83 \\
\hline & $\begin{array}{l}\text { DAT availability might be different between motor subtypes in relation to different disease } \\
\text { severity; striatal dopamine depletion may also contribute to both cognitive (ie, executive tasks) } \\
\text { and nonmotor symptoms (ie, anxiety) in de novo PD patients. }\end{array}$ & $87-89$ \\
\hline \multirow{4}{*}{$\begin{array}{l}\text { Magnetic resonance imaging } \\
\text { (MRI) }\end{array}$} & Neuromelanin decrease (whose paramagnetic properties result in high signal on specific & 84 \\
\hline & $\begin{array}{l}\text { TI-weighted sequences) in the substantia nigra and locus coeruleus may differentiate } \\
\text { between PD patients and healthy controls. }\end{array}$ & \\
\hline & $\begin{array}{l}\text { Functional MRI can discriminate among PD patients with TD and non-TD phenotype and } \\
\text { healthy controls. }\end{array}$ & 85 \\
\hline & $\begin{array}{l}\text { MRI shows positive correlation between TD phenotype and transverse relaxation rate } \\
\text { (reflecting iron load) within the putamen, caudate, and thalamus. }\end{array}$ & 86 \\
\hline
\end{tabular}

Abbreviations: BDNF, brain-derived neurotrophic factor; IGF, insulin-like growth factor; CSF, cerebrospinal fluid; PD, Parkinson's disease; PDGFB, platelet-derived growth factor subunit B; TD, tremor dominant; RBD, REM-sleep behavior disorders; REM, rapid eye movement; GH, growth hormone; $A \beta$, amyloid beta.

sleep behavior disorders, RBD), excessive daytime sleepiness, and postprandial fullness. Interestingly, constipation, frequent nightmares, and dream-enacting behavior were found to associate together across all premotor time spans. ${ }^{3}$

A recent systematic meta-analysis showed that positive familial history for either PD or tremor, constipation, and lack of smoking history were strongly associated with later development of PD. ${ }^{4}$ In accordance with these data, a survey among 116 de novo PD patients and 232 controls showed that current smokers were less likely to have PD, while former smokers were more likely to have PD, as compared to never-smokers, further suggesting that quitting smoking may be considered an early NMS of PD. ${ }^{5}$

Further, in agreement with other data, ${ }^{6-8}$ a recent population-based study demonstrated that at 5 years before diagnosis, patients who went on to develop PD had a higher incidence of tremor, balance impairments, constipation, hypotension, erectile dysfunction, urinary dysfunction, dizziness, fatigue, depression, and anxiety compared with controls. At 10 years before diagnosis of PD, the incidence of tremor and constipation was higher in those who went on to develop PD than in controls. ${ }^{9}$ According to prospective 
Table 3 Biomarkers for PD progression

\begin{tabular}{|c|c|c|}
\hline Biomarker & Comments & References \\
\hline \multicolumn{3}{|l|}{ Clinical biomarkers } \\
\hline \multirow[t]{2}{*}{ Non-motor symptoms (NMS) } & NMS tend to remain stable during the early phase of disease. & 97 \\
\hline & $\begin{array}{l}\text { Several clinical biomarkers herald worsening of cognition, as the presence of } \mathrm{MCl} \text {, apathy, } \\
\text { subjective memory complaints, and visual hallucinations. }\end{array}$ & $98-103$ \\
\hline \multirow[t]{2}{*}{ Motor symptoms } & $\begin{array}{l}\text { TD variant has a slower disease progression rate, less cognitive decline, and lower incidence } \\
\text { of neuropsychiatric complications such as visual hallucinations and depression, when } \\
\text { compared to the non-TD phenotype. }\end{array}$ & 37,91 \\
\hline & Freezing of gait may represent a biomarker for development of cognitive impairment and dementia. & $93-96$ \\
\hline \multicolumn{3}{|l|}{ Biochemical biomarkers } \\
\hline Metabolic factors & The overall cardiovascular risk has been associated with axial motor impairment. & 105 \\
\hline \multirow[t]{4}{*}{ Neurotrophic factors } & $\begin{array}{l}\text { Serum BDNF levels have been associated with the severity of PD motor symptoms } \\
\text { and cognitive symptoms. }\end{array}$ & 45,106 \\
\hline & $\begin{array}{l}\text { Serum epidermal growth factor (EGF) levels have been associated with cognitive deficits } \\
\text { within } 2 \text { years since diagnosis. }\end{array}$ & 107,108 \\
\hline & IGF-I levels have been associated with both motor and cognitive symptoms. & 48,49 \\
\hline & $\begin{array}{l}\text { Vitamin D levels were negatively associated with motor disability, cognitive functions, } \\
\text { and psychiatric symptoms. }\end{array}$ & 50,109 \\
\hline \multirow[t]{3}{*}{ Oxidative stress } & Coenzyme Q10 levels are inversely associated with motor symptoms. & 113 \\
\hline & Higher bilirubin levels were associated with worse motor symptoms. & 54 \\
\hline & Higher UA levels were associated with less motor and non-motor burden. & $114-123$ \\
\hline \multirow[t]{2}{*}{ Iron and other metals } & $\begin{array}{l}\text { Higher iron levels have been associated with the presence of RBD, poor quality of sleep, } \\
\text { and depression. }\end{array}$ & $125-127$ \\
\hline & Serum manganese and copper have been associated with depressive symptoms. & 127 \\
\hline \multirow[t]{4}{*}{ CSF biomarkers } & Conflicting results have been reported when analyzing the relationship between & $71,72,128,129$ \\
\hline & $\alpha$-synuclein and motor severity of PD. & \\
\hline & Higher phosphorylated-tau protein has been related with greater cognitive decline. & $|30,13|$ \\
\hline & $\begin{array}{l}\text { CSF A } \beta \text { peptides have also been studied in relation to cognitive functions in early PD, } \\
\text { but conflicting results have been reported. }\end{array}$ & 68,69 \\
\hline \multicolumn{3}{|l|}{ Imaging biomarkers } \\
\hline \multirow{4}{*}{$\begin{array}{l}\text { Magnetic resonance imaging } \\
\text { (MRI) }\end{array}$} & Functional MRI shows resting-state functional connectivity changes in relation to cognitive decline & 132,133 \\
\hline & $\begin{array}{l}\text { Gray matter volume analysis suggest that the caudate volume loss may contribute to cognitive } \\
\text { decline while changes in thalamic volume may have relevance to tremor severity. }\end{array}$ & 135 \\
\hline & $\begin{array}{l}\text { A positive correlation between Mini-Mental State Examination scores and cortical thickness in } \\
\text { the anterior temporal, dorsolateral prefrontal, posterior cingulate, temporal fusiform, and } \\
\text { occipito-temporal cortex has been detected supporting the usefulness of cortical thickness } \\
\text { measurement in assessing disease stage and cognitive impairment in patients with PD. }\end{array}$ & 136,137 \\
\hline & $\begin{array}{l}\text { Iron content in basal ganglia, assessed as MRI relaxation rate } \mathrm{R} 2^{*} \text {, has been proposed as } \\
\text { a biomarker of PD progression in PD. }\end{array}$ & 138 \\
\hline
\end{tabular}

Abbreviations: CSF, cerebrospinal fluid; IGF-I, insulin-like growth factor-I; PD, Parkinson's disease; TD, tremor dominant; MCI, mild cognitive impairment; BDNF, brainderived neurotrophic factor; UA, uric acid; RBD, REM-sleep behavior disorders; REM, rapid eye movement; $A \beta$, amyloid beta, R2*, effective transverse relaxation rate.

studies, RBD and impaired olfaction are, by far, associated with the highest PD risk. ${ }^{10-12}$

The occurrence of NMS during the premotor phase reflects the widespread neurochemical and neuroanatomical changes that occur throughout the course of PD, with involvement of not only the dopaminergic nigrostriatal system but also serotonergic and noradrenergic brain stem areas, cholinergic frontal and brain stem regions. ${ }^{13}$ However, each of these clinical biomarkers has poor predictive value when considered individually. Thus, ideally, screening strategies should include a combination of clinical and imaging as well as biochemical biomarkers to increase the likelihood of diagnosing as many individuals (developing PD) as possible (see "Biochemical biomarkers").

\section{Biochemical biomarkers}

Metabolic factors

Conflicting data suggest that increase in total cholesterol may be either a protective or a risk factor for PD. ${ }^{14,15}$ Indeed, the association with motor symptoms may be explained in light of the white matter damage associated with metabolic factors, although a direct effect on neurodegenerative process has been hypothesized. 


\section{Neurotrophic factors}

Insulin-like growth factor 1 (IGF-1) has been shown to provide effective protection against the loss of dopaminergic neurons in cellular and animal models of PD. Preliminary studies found that IGF-1 levels were directly associated with the burden of motor dysfunction in subjects at risk of PD. ${ }^{16,17}$

Vitamin D may exert a neuroprotective activity, although the exact mechanism is not fully understood. Accordingly, a 30-year longitudinal Finnish study showed that individuals with higher serum vitamin D concentrations have a $30 \%$ reduced risk of PD. ${ }^{18}$

\section{Neuroinflammation}

Neuroinflammation indeed play a role in enhancing neurodegenerative processes. However, there are no studies on single markers of neuroinflammation.

\section{Oxidative stress}

Oxidative stress plays a significant role in PD pathogenesis. Uric acid (UA) has antioxidant and iron scavenger features, possibly providing natural neuroprotection against PD. Evidence suggests a protective role of higher UA levels ${ }^{19-21}$ and of gout in the development of PD. ${ }^{22,23}$

\section{CSF biomarkers}

To our knowledge, no study has investigated possible cerebrospinal fluid (CSF) biomarkers for prodromal phase of PD to date.

\section{Imaging biomarkers}

It is unrealistic to expect that a single biomarker will fulfill all the criteria for a reliable prodromal marker of PD. Indeed, using a combination of tools merging clinical and imaging biomarkers is the most likely rational approach. Different techniques have been used to visualize striatal or nigrostriatal denervation with nuclear imaging (both positron emission tomography [PET] and single-photon emission computed tomography [SPECT]) or the hyperechogenicity of the substantia nigra ( $\mathrm{SN}$ ) with transcranial sonography (TCS), while functional magnetic resonance imaging (fMRI) has been used to visualize abnormalities in corticostriatal circuits.

The Parkinson-Associated Risk Syndrome (PARS) study applied a two-stage screening strategy for PD consisting of olfactory testing followed by dopamine transporter (DaT) imaging (DaTscan) to detect nigrostriatal degeneration. ${ }^{24,25}$ Among the 5,000 individuals screened with the 40-item University of Pennsylvania Smell Identification Test (UPSIT), 669 were at or below the 15th percentile based on age and sex, indicating hyposmia. ${ }^{25}$ Among those, 303 (203 hyposmic and 100 normosmic) were subjected to DaTscan, and nigrostriatal dysfunction was detected in $11 \%$ of the hyposmic subjects compared with $1 \%$ of the normosmic subjects. Combining multiple factors (hyposmia, male sex, and constipation) increased the probability of detecting subjects with a nigrostriatal deficit, up to $40 \% .^{26}$

TCS is an imaging technique based on reflection and scattering of ultrasound waves at interfaces with diverse acoustic impedance depicting the brain as the mesencephalon and the basal ganglia. The alteration in SN signal, seen as an area of increased signal intensity and extent (termed hyperechogenicity), has been proposed as an easy and noninvasive biomarker of PD. ${ }^{27,28}$ The Prospective Validation of Risk factors for the development of Parkinson Syndromes (PRIPS) study showed that enlarged SN hyperechogenicity is a good biomarker of PD development at both 3-27 and 5-year follow-up. ${ }^{28}$ However, another prospective study showed low sensitivity and specificity of TCS in predicting conversion to PD in patients affected by idiopathic RBD when used alone. ${ }^{29}$ The Sleep Innsbruck Barcelona group showed that the combination of ioflupane $\left({ }^{123} \mathrm{I}\right)$ single photon emission computed tomography ([23I]-FP-CIT SPECT) and TCS can better predict conversion to synucleinopathy in idiopathic RBD. ${ }^{30}$

Healthy carriers of the G2019S LRRK2 mutation display a markedly increased, age-dependent risk of developing PD and, thus, represent an ideal enriched cohort to test the feasibility of biomarkers' screening approaches. Both TCS and fMRI show specific alterations in these individuals prior to PD diagnosis. ${ }^{31,32}$

\section{Biomarkers for diagnosis Clinical biomarkers}

Currently, diagnosis of PD is based on the presence and combination of cardinal motor signs..$^{33}$ However, increasing evidence suggests a major role for a complex spectrum of NMS in the characterization of PD since the onset, possibly helpful in the process of redefinition of PD diagnosis. ${ }^{12}$

The PRIAMO (PaRkInson And non-Motor symptOms) study first showed that early, drug-naïve patients complained more frequently about psychiatric disturbances (66\%), fatigue (52\%), and attention/memory problems (48\%). ${ }^{34}$ Another study showed that both RBD and insomnia were associated with lower scores on several cognitive tests in early, drug-naïve patients possibly suggesting that sleep symptoms might be considered an early marker of dementia. ${ }^{35} \mathrm{~A}$ survey examining the prevalence of NMS at onset in 200 PD patients and 93 healthy controls showed specific sex-related differences in the 
spectrum of NMS with men with PD complaining of problems having sex and taste/smelling difficulties significantly more frequently than women with PD. ${ }^{36}$

From a purely motor perspective, it is possible to distinguish two main distinct clinical subtypes in consideration of the predominant motor features, a tremor dominant (TD) and an akinetic-rigid type (ART), and one mixed phenotype, not presenting one prevailing motor feature. ${ }^{37}$ In keeping with the increasing role of NMS, more recent approaches provide classification of PD patients at diagnosis based on both motor and non-motor features. ${ }^{38}$ A study performing a cluster analysis using data assessing both motor and NMS with a data-driven approach in 100 newly diagnosed untreated PD patients identified four distinct groups of patients, namely (1) benign pure motor, (2) benign mixed motor-non-motor, (3) non-motor dominant, and (4) motor dominant, suggesting the presence of a cross talk between motor and NMS in defining PD phenotype at diagnosis. As a matter of fact, the identification of such subtypes may have important implications for generating pathogenetic hypotheses and therapeutic strategies. ${ }^{39}$

Newer and even more recent approach for PD subtyping encompasses clinical and genetic data. ${ }^{40} \mathrm{~A}$ population-based modeling study proposed such a model which correctly distinguished PD patients from healthy controls in the Parkinson's Progression Markers Initiative with high sensitivity and specificity. ${ }^{40}$ Further application of this model to premotor prospective cohorts could facilitate identification of biomarkers and interventions. ${ }^{40}$

Another role for clinical biomarkers of diagnosis is to help differentiating PD from atypical parkinsonism, as multiple system atrophy (MSA). Besides the poor response to levodopa, and the additional presence of pyramidal or cerebellar signs or autonomic failure as major diagnostic criteria, certain other clinical features known as "red flags" or warning signs may raise the clinical suspicion of MSA. ${ }^{41}$

\section{Biochemical biomarkers} Metabolic factors

Homocysteine is a neurotoxic, intermediary product of the methionine cycle, whose concentrations can be influenced by different factors and, in particular, by the genetic background. ${ }^{42}$ Interestingly, homocysteine and its metabolites (ie, methylmalonate or cystatin $\mathrm{C}$ ) have been suggested to be increased in PD, since the time of the diagnosis. ${ }^{43,44}$

\section{Neurotrophic factors}

Preclinical evidence shows that the brain-derived neurotrophic factor takes part in the survival and in the activity of striatal dopaminergic neurons. Accordingly, serum BDNF levels have been found to be decreased in PD patients as compared to controls ${ }^{45}$ and associated with striatal DaT binding. ${ }^{46}$

Growth hormone (GH) and growth hormone-inhibiting hormone (or somatostatin) pathways have been investigated as diagnostic markers of PD. In particular, the growth hormone response to arginine test may be useful to differentiate PD from MSA. ${ }^{47}$

IGF-1 has been reported to be higher in PD patients than in controls ${ }^{16,48,49}$ with one single study suggesting that a cutoff value of $114 \mathrm{ng} / \mathrm{mL}$ might correctly differentiate de novo PD patients from controls. ${ }^{16}$

PD patients have lower serum vitamin D levels as compared to controls. ${ }^{50}$ Given the fact that seasonal differences in vitamin D levels have been observed, ${ }^{50}$ one study suggested that the season of birth is a predictor of PD development. ${ }^{51}$

\section{Neuroinflammation}

Platelet-derived growth factor subunit B and prolactin as well as other neuroinflammatory markers have been reported as significantly different between PD patients and controls. ${ }^{52,53}$ However, conflicting results have been reported so far, possibly because of differences in methodologies applied for determining these markers and of heterogeneity in populations.

\section{Oxidative stress}

Bilirubin levels were reported to be either increased ${ }^{54}$ or decreased $^{55}$ in PD patients compared to healthy controls. Overall, PD populations that have been described so far are heterogeneous, and these conflicting results might be explained by the presence of confounding factors affecting bilirubin levels and/or by bilirubin variations during the course of the disease.

Decreased serum ceruloplasmin levels might reflect more severe oxidative stress with subsequent neurodegenerative alterations. Accordingly, lower serum ceruloplasmin levels have been associated with younger age at onset of $\mathrm{PD},{ }^{56}$ and with more impaired neuroimaging features within the $\mathrm{SN} .{ }^{57}$

Several meta-analyses confirm that PD patients, with particular regard to men, present lower UA levels as compared to controls, notwithstanding potential confounding factors (ie, body mass index or comorbidities). ${ }^{58,59}$ Interestingly, higher UA levels were associated with the likelihood of having a scan without evidence of dopaminergic deficit in a secondary analysis of the PRECEPT study (recruiting 
800 early PD), ${ }^{60}$ and with an increased DaT availability among newly diagnosed PD patients, suggesting that neurodegenerative alterations can be tracked based on UA levels right from the early phases of the disease. ${ }^{61}$

\section{Iron and other metals}

Each positive variation of $10 \mu \mathrm{g} / \mathrm{dL}$ of serum iron has been associated with 3\%-10\% reduced relative risk of having $\mathrm{PD},{ }^{62}$ suggesting the involvement of iron metabolism in PD pathogenesis. ${ }^{62}$ Among other metals, manganese (Mn) has been associated with PD. ${ }^{63}$

\section{$\alpha$-Synuclein}

Lewy bodies, the neuropathological hallmark of PD, are mainly composed of $\alpha$-synuclein ( $\alpha$-syn), and, thus, different studies have investigated $\alpha$-syn as a diagnostic biomarker in serum and plasma. ${ }^{64,65} \mathrm{It}$ is still debated if plasmatic levels of $\alpha$-syn are similar, ${ }^{66}$ or higher in $\mathrm{PD}$, as compared to healthy controls, when there is an increased efflux of the protein to the peripheral blood of these patients. ${ }^{67}$ However, utilization of different methods to detect $\alpha$-syn in plasma possibly account for such divergent results.

\section{CSF biomarkers}

CSF amyloid beta peptides are altered from the early phases of PD, ${ }^{68,69}$ especially in patients with dementia. ${ }^{70}$ Regarding $\alpha$-syn, the majority of recent studies have shown decreased CSF levels in PD patients, as compared to controls, although this marker apparently cannot distinguish among different synucleopathies. ${ }^{71-73}$ In addition, levels of CSF tau protein were higher in PD patients, in particular in the early phases, as compared to controls. ${ }^{74}$

Among other CSF biomarkers, DJ-1 levels were found to increase in early $\mathrm{PD}$ patients compared to controls. ${ }^{75}$ However, CSF DJ-1 increased levels are not specific and, for instance, they are also raised in MSA. ${ }^{76}$ Furthermore, several studies suggest an increase in the number of CSF biomarkers with regard to the status of oxidative balance in PD patients when compared to controls. ${ }^{77,78}$

Different studies have been investigating difference in CSF metabolomics between PD patients and controls. In particular, metabolomics focuses on the quantitative analysis of the small metabolites affected by PD. Preliminary data show that specific combinations of metabolites can differentiate PD patients from healthy controls. ${ }^{79,80}$ Overall, these studies provide a significant contribution in the identification of novel biomarkers that enable early diagnosis of the disease, as well as provide knowledge about the metabolic pathways and molecular mechanisms involved.

\section{Imaging biomarkers}

Clinically, there is no indication to perform imaging studies when the patient fulfills PD clinical criteria ${ }^{33}$ and there is no diagnostic doubt. On the other hand, imaging may be sometimes helpful in the differential diagnosis with other movement disorders (ie, essential tremor and atypical parkinsonism). However, when it comes to research, imaging biomarkers may be helpful in further delineating the different PD phenotypes at diagnosis.

Evaluation of the presynaptic dopaminergic function by means of either 6-[18F] fluoro-L-dopa or VMAT 2 or DaT availability measurement can be helpful in differentiating degenerative parkinsonism from essential tremor (eg, DaT availability is approved by the Food and Drug Administration for this purpose), ${ }^{81}$ and secondary parkinsonism, but it is not helpful in the differential diagnosis with atypical parkinsonism. For this purpose, it may be useful to combine pre- and postsynaptic dopaminergic imaging (with PET tracers for D2-like dopamine receptors), or to use fluorodeoxyglucosePET which may disclose specific metabolic patterns for the different forms of atypical parkinsonism. ${ }^{82}$

As sympathetic denervation in PD involves postganglionic neurons, and MSA affects the preganglionic neurons, cardiac $\left[{ }^{123} \mathrm{I}\right]$ metaiodobenzylguanidine scintigraphy may also be useful for the differential diagnosis of these disorders. ${ }^{83}$

Recent studies have suggested that neuromelanin decrease (whose paramagnetic properties result in high signal on specific T1-weighted magnetic resonance imaging [MRI]) in the SN and locus coeruleus of PD patients may differentiate between PD patients and healthy controls with high sensitivity and specificity. ${ }^{84}$

For a research purpose, imaging studies may further contribute in the delineation of different PD phenotypes at diagnosis. A recent fMRI study demonstrated that the subtype-specific functional networks could discriminate among PD patients with TD and non-TD phenotypes and healthy controls. ${ }^{85}$ Further supporting different imaging features according to motor phenotype, another MRI study detecting iron load found a positive correlation between TD phenotype and transverse relaxation rate (reflecting iron load) within the putamen, caudate, and thalamus. ${ }^{86}$

Nuclear imaging studies also suggest different patterns of uptake of radiotracers according to motor phenotype. A recent study investigating the role of presynaptic nigrostriatal dopaminergic damage in 51 drug-naïve $\mathrm{PD}$ patients with 
ART and TD phenotypes found that ART patients presented higher motor scores and lower DaT availability in affected and unaffected putamen, suggesting that $\mathrm{DaT}$ availability might be different between motor subtypes in relation to different regimens of disease severity. ${ }^{87}$

Nuclear tracers may also present different patterns in relation to cognitive profile and NMS at PD diagnosis. Recent data suggest that striatal dopamine depletion may contribute to both cognitive (ie, executive tasks) ${ }^{88}$ and NMS (ie, anxiety) ${ }^{89}$ in de novo PD patients.

\section{Biomarkers of progression Clinical biomarkers}

Identifying those patients at risk of greater progression (eg, with development of cognitive impairment) is of particular importance in order to include patients in clinical trials when DMT are available.

Based on pathological evidence, ${ }^{90}$ clinical studies showed that the TD variant has a slower disease progression, less cognitive decline, and lower incidence of neuropsychiatric complications such as visual hallucinations and depression, when compared to the ART group. ${ }^{37,91}$

Although dopaminergic treatment may mask disease progression, reported evidence suggests that several symptoms become severe with disease duration. However, little is known about the anatomical progression over the body segments of extrapyramidal signs in PD. Furthermore, a significant unmet need is the availability of instruments to detect disease progression, even in the early phase and on dopaminergic medications. ${ }^{92}$

Motor symptoms, as freezing of gait, may represent as a biomarker of development of cognitive impairment and dementia. ${ }^{93,94}$ Indeed, gait is not merely considered a pure motor task as evidence shows the influence of specific cognitive profiles on gait parameters suggesting the existence of a complex interplay between gait and cognition..$^{95,96}$

NMS generally tend to remain stable during the early phase of disease, with only a few of them being affected by dopaminergic therapy and, specifically, by the use of dopamine agonists (ie, reduction in depression and increase in weight). ${ }^{97,98}$ However, several clinical biomarkers have shown to herald worsening of cognition in PD patients, as the presence of mild cognitive impairment, ${ }^{99,100}$ apathy, ${ }^{101}$ subjective memory complaints, ${ }^{102}$ and visual hallucinations. ${ }^{103}$ Interestingly, a recent study suggested that voluptuary habits might also affect the burden of NMS and, for instance, smoking might be associated with sleep disturbances and sexual difficulties. ${ }^{104}$

\section{Biochemical biomarkers}

\section{Metabolic factors}

The overall cardiovascular risk has been associated with the rate of accrual of axial motor impairment, ${ }^{105}$ possibly by determining white matter changes.

\section{Neurotrophic factors}

Brain-derived neurotrophic factor serum levels were found to be associated with the severity of PD motor symptoms and cognitive symptoms. ${ }^{45,106}$ Overall, these findings suggest that brain-derived neurotrophic factor serum levels may represent a biomarker not only of motor and cognitive dysfunction in PD but also of the effects of treatments on these outcomes.

Epidermal growth factor acts as a neurotrophic factor on dopaminergic nigrostriatal neurons in animal models of PD. Confirming previous data, ${ }^{107}$ Pellecchia et a ${ }^{108}$ showed the association between levels of epidermal growth factor, measured at the time of PD diagnosis and cognitive deficits within 2-year follow-up, with particular regard to executive dysfunctions.

Intriguingly, Picillo et $\mathrm{a}^{48}$ suggested that IGF-1 levels might be associated with those motor symptoms predominantly responsive to dopaminergic treatment, as compared to the whole UPDRS-III score, since IGF-1 seems to act specifically on dopaminergic cells. With regard to NMS, IGF-1 levels were positively associated with cognitive functions and, in particular, with executive and memory tasks. ${ }^{49}$

Vitamin D levels were negatively associated with motor disability, ${ }^{50,109}$ with cognitive functions (in particular with executive and memory tasks), and with psychiatric symptoms.

\section{Neuroinflammation}

Several neuroinflammatory factors were associated with motor and NMS in PD. ${ }^{110-112}$ However, these results are based on small samples and need to be replicated in different population in order to test their reliability.

\section{Oxidative stress}

Coenzyme Q10 is a powerful antioxidant whose levels are inversely associated with motor symptoms of PD, thus supporting its therapeutic use. ${ }^{113}$

Higher bilirubin levels were associated with worse motor symptoms at the time of PD diagnosis in a cohort of 75 de novo patients, but with better ones after 2-year follow-up, suggesting that higher bilirubin levels might depict a more pronounced neurodegenerative process, but are possibly related to improved outcomes over time. ${ }^{54}$ 
Higher UA levels were associated with slower motor progression and with a reduced risk of requiring levodopa treatment, in a secondary analysis of the DATATOP study. ${ }^{114}$ The latter result has been confirmed in different studies conducted on de novo or advanced PD populations. ${ }^{115-117}$ It is worth noting that UA levels might be particularly lower in those subjects with predominant axial features, as compared to TD patients, suggesting a more severe disease course. ${ }^{118}$ Accordingly, higher UA levels have been associated with $80 \%$ reduced likelihood of wearing-off ${ }^{119}$ and with lower total score of the NMS questionnaire. In detail, UA levels have been associated with specific NMS, such as attention/ memory, depression/anxiety, and cardiovascular. ${ }^{17,120,121}$ The association with the impairment of attention/memory domains seems particularly promising since confirmed by different studies exploring cognitive function with comprehensive neuropsychological batteries. ${ }^{122,123}$ Furthermore, a recent Phase 2 trial on the use of inosine in PD, a precursor of UA, showed a possible association between increased UA levels and mood disorders assessed with the Geriatric Depression Scale. ${ }^{124}$

\section{Iron and other metals}

Iron metabolism has been associated with the presence of RBD and, more in general, with poor quality of sleep in PD patients. ${ }^{125,126}$ Furthermore, there is preliminary evidence of higher iron levels in PD patients with depression. ${ }^{127}$

Manganese and copper have also been associated with depressive symptoms of $\mathrm{PD}$, but these findings need to be confirmed by further studies on larger samples. ${ }^{127}$

\section{CSF biomarkers}

Conflicting results have been reported when analyzing the possible relationship between $\alpha$-syn and motor severity of PD. ${ }^{71,128,129}$ In a secondary analysis of the DATATOP study, lower baseline CSF $\alpha$-syn levels predicted a better preservation of cognitive functions in early PD patients after 8 -year-follow-up. ${ }^{72}$

Another analysis of the DATATOP study found an association between higher phosphorylated-tau protein and greater decline in cognitive functions but slower motor progression. ${ }^{130,131}$ Thus, it is clear that the role of tau protein is largely unknown, and, so far there are only association studies, not providing possible explanations on the importance of tau species in PD pathogenesis.

CSF amyloid beta peptides have also been studied in relation to cognitive functions in early $\mathrm{PD}$, but conflicting results have been reported. ${ }^{68,69}$

\section{Imaging biomarkers}

Once the overt disease has become manifested, imaging biomarkers of prodromal or diagnosis of PD (ie, TCS, DaT imaging, etc) do not reliably feature clinical disease progression and, thus, are not considered useful biomarkers of progression. Thus, other imaging markers are currently being tested and validated as biomarkers of disease progression and many other in this regard are in the pipeline.

A study evaluating fMRI whole-brain resting-state functional connectivity changes in relation to cognitive decline found a decrease in functional connectivity in PD patients independent of aging effects and directly related to cognitive decline. ${ }^{132}$ These findings confirm previous data, ${ }^{133}$ and support the role of reduced functional connectivity in cognitive decline and dementia of PD. ${ }^{132}$

Previous evidence showed a resting state functional connectivity disruption of "executive attention" and visual neural networks in association with the presence of freezing of gait in PD. ${ }^{134}$ Indeed, this data further strengthened the relationship between gait and cognition in PD. ${ }^{134}$

PD pathology leads to volumetric changes in the brain. A study investigated the pattern of gray matter changes according to disease progression in 89 patients with $\mathrm{PD}$ and detected decreased gray matter volume in the fronto-temporoparietal areas and the bilateral caudate and increased gray matter volume in the bilateral limbic/paralimbic areas, medial globus pallidus/putamen, and the right occipital cortex as compared with healthy controls. This study suggested that the caudate volume loss may contribute to cognitive decline, while changes in thalamic volume may have relevance to tremor severity. ${ }^{135}$

Supporting previous findings, ${ }^{136}$ a recent study found a positive correlation between Mini-Mental State Examination scores and cortical thickness in the anterior temporal, dorsolateral prefrontal, posterior cingulate, temporal fusiform, and occipito-temporal cortex, thus supporting the usefulness of cortical thickness measurement in assessing disease stage and cognitive impairment in patients with PD. ${ }^{137}$

Even more recently, changes in iron content in basal ganglia, assessed as MRI relaxation rate R2*, have been proposed as a biomarker of disease progression in $\mathrm{PD}$, suggesting that R2* MRI could be an interesting tool for individual assessment of neurodegeneration, and also be useful in testing the efficiency of DMT. ${ }^{138}$

\section{Conclusion}

In this review, we have summarized the current insights on clinical, imaging, and biochemical biomarkers as markers 
for prodromal phase, diagnosis, and disease progression of PD. Indeed, it is unrealistic to expect that there will be a unique biomarker useful to discriminate between PD and other diseases with high sensitivity and specificity as well as to track progression throughout the entire disease course. ${ }^{139}$ By combining microscopic (CSF $\alpha$-syn) and macroscopic (fMRI functional connectivity) observations, as well as Pittsburgh compound B-PET imaging to detect amyloid beta peptides' deposit, Campbell et al ${ }^{140}$ provided a first step toward a comprehensive combined biomarkers strategy. In this cross-sectional study, the authors measured soluble CSF $\alpha$-syn in PD patients without dementia and found that reduced CSF $\alpha$-syn levels correlated with reduced fMRI sensorimotor connectivity. Indeed, the current challenge as well as the future perspective of development of biomarkers in PD is to find the best combination of biomarkers to track the disease progression since the prodromal stages.

In this context, genetics as well as the possibility to share data through consortia will accelerate the pace of PD biomarker research. ${ }^{40,141}$

\section{Author contributions}

MP contributed to conception, organization, and execution of the research project; manuscript writing of the first draft. MM contributed to organization and execution of the research project, manuscript writing of the first draft. ES contributed to execution of the research project, manuscript writing of the first draft. MTP contributed to conception and organization of the research project, review and critique the manuscript. MP, MM, ES, and MTP gave final approval of the version to be published. All authors contributed toward data analysis, drafting and critically revising the paper and agree to be accountable for all aspects of the work.

\section{Disclosure}

MP, PB, and MTP were staff at the University of Salerno, Italy. MM and ES were staff at the "Federico II" University of Naples, Italy. The authors did not receive financial support for this research. All expenses were funded by the researchers. The authors report no other conflicts of interest in this work.

\section{References}

1. Biomarkers Definitions Working Group. Biomarkers and surrogate endpoints: preferred definitions and conceptual framework. Clin Pharmacol Ther. 2001;69:89-95.

2. Wright-Willis A, Evanoff BA, Lian M, Criswell SR, Racette BA. Geographic and ethnic variation in Parkinson disease: a populationbased study of US Medicare beneficiaries. Neuroepidemiology. 2010;34(3):143-151.
3. Pont-Sunyer C, Hotter A, Gaig C, et al. The onset of nonmotor symptoms in Parkinson's disease (the ONSET PD study). Mov Disord. 2015;30(2):229-237.

4. Noyce AJ, Bestwick JP, Silveira-Moriyama L, et al. Meta-analysis of early nonmotor features and risk factors for Parkinson disease. Ann Neurol. 2012;72(6):893-901.

5. Moccia M, Erro R, Picillo M, et al. Quitting smoking: an early nonmotor feature of Parkinson's disease? Parkinsonism Relat Disord. 2015;21(3):216-220.

6. Schrag A, Horsfall L, Walters K, Noyce A, Petersen I. Prediagnostic presentations of Parkinson's disease in primary care: a case-control study. Lancet Neurol. 2015;14(1):57-64.

7. Wu YH, Liao YC, Chen YH, Chang MH, Lin CH. Risk of premotor symptoms in patients with newly diagnosed PD: a nationwide, population-based, case-control study in Taiwan. PLoS One. 2015; 10(6): 0130282.

8. Shen CC, Tsai SJ, Perng CL, Kuo BI, Yang AC. Risk of Parkinson disease after depression: a nationwide population-based study. Neurology. 2013;81(17):1538-1544.

9. Adams-Carr KL, Bestwick JP, Shribman S, Lees A, Schrag A, Noyce AJ. Constipation preceding Parkinson's disease: a systematic review and meta-analysis. J Neurol Neurosurg Psychiatry. Epub 2015 Sep 7.

10. Schenck CH, Boeve BF, Mahowald MW. Delayed emergence of a parkinsonian disorder or dementia in $81 \%$ of older males initially diagnosed with idiopathic REM sleep behavior disorder: 16-year update on a previously reported series. Sleep Med. 2013;14(8):744-748.

11. Iranzo A, Tolosa E, Gelpi E, Molinuevo JL, Valldeoriola F, Serradell $\mathrm{M}$, et al. Neurodegenerative disease status and post-mortem pathology in idiopathic rapid-eye-movement sleep behaviour disorder: an observational cohort study. Lancet Neurol. 2013;12(5):443-453.

12. Berg D, Marek K, Ross GW, Poewe W. Defining at-risk populations for Parkinson's disease: lessons from ongoing studies. Mov Disord. 2012;27(5):656-665.

13. O'Sullivan SS, Williams DR, Gallagher DA, Massey LA, SilveiraMoriyama L, Lees AJ. Nonmotor symptoms as presenting complaints in Parkinson's disease: a clinicopathological study. Mov Disord. 2008;23(1):101-106.

14. Hu G. Total cholesterol and the risk of Parkinson's disease: a review for some new findings. Parkinsons Dis. 2010;2010:836962.

15. De Lau LML, Koudstaal PJ, Hofman A, Breteler MMB. Serum cholesterol levels and the risk of Parkinson's disease. Am J Epidemiol. 2006;164(10):998-1002.

16. Godau J, Herfurth M, Kattner B, Gasser T, Berg D. Increased serum insulin-like growth factor 1 in early idiopathic Parkinson's disease. J Neurol Neurosurg Psychiatry. 2010;81(5):536-538.

17. Godau J, Knauel K, Weber K, et al. Serum insulinlike growth factor 1 as possible marker for risk and early diagnosis of Parkinson disease. Arch Neurol. 2011;68(7):925-931.

18. Knekt P, Kilkkinen A, Rissanen H, Marniemi J, Sääksjärvi K, Heliövaara M. Serum vitamin D and the risk of Parkinson disease. Arch Neurol. 2010;67(7):808-811.

19. de Lau L, Koudstaal P, Hofman A, Breteler M. Serum uric acid levels and the risk of Parkinson disease. Ann Neurol. 2005;585:797-800.

20. Weisskopf M, O'Reilly E, Chen H, Schwarzschild M, Ascherio A. Plasma urate and risk of Parkinson's disease. Am J Epidemiol. 2007;166(5):561-567.

21. Chen H, Mosley T, Alonso A, Huang X. Plasma urate and Parkinson's disease in the Atherosclerosis Risk in Communities (ARIC) study. Am J Epidemiol. 2009;169(9):1064-1069.

22. Alonso A, Rodríguez L, Logroscino G, Hernán M. Gout and risk of Parkinson disease: a prospective study. Neurology. 2007;69(17):1696-1700.

23. De Vera M, Rahman M, Rankin J, Kopec J, Gao X, Choi H. Gout and the risk of Parkinson's disease: a cohort study. Arthritis Rheum. 2008;59(11):1549-1554.

24. Stern MB, Siderowf A. Parkinson's at risk syndrome: can Parkinson's disease be predicted? Mov Disord. 2010;25 Suppl 1:S89-93. 
25. Siderowf A, Jennings D, Eberly S, et al. Impaired olfaction and other prodromal features in the Parkinson at-risk syndrome study. Mov Disord. 2012;27(3):406-412.

26. Jennings D, Siderowf A, Stern M, et al. Imaging prodromal Parkinson disease: the Parkinson associated risk syndrome study. Neurology. 2014;83(19):1739-1746.

27. Berg D, Seppi K, Behnke S, et al. Enlarged substantia nigra hyperechogenicity and risk for Parkinson disease: a 37-month 3-center study of 1847 older persons. Arch Neurol. 2011;68(7):932-937.

28. Berg D, Behnke S, Seppi K, et al. Enlarged hyperechogenic substantia nigra as a risk marker for Parkinson's disease. Mov Disord. 2013;28(2):216-219.

29. Iranzo A, Stockner H, Serradell M, et al. Five-year follow-up of substantia nigra echogenicity in idiopathic REM sleep behavior disorder. Mov Disord. 2014;29(14):1774-1780.

30. Iranzo A, Valldeoriola F, Lomeña F, et al. Serial dopamine transporter imaging of nigrostriatal function in patients with idiopathic rapid-eyemovement sleep behaviour disorder: a prospective study. Lancet Neurol 2011;10(9):797-805.

31. Vilas D, Ispierto L, Álvarez R, et al. Clinical and imaging markers in premotor LRRK2 G2019S mutation carriers. Parkinsonism Relat Disord. 2015;21(10):1170-1176.

32. Helmich RC, Thaler A, van Nuenen BF, et al. Reorganization of corticostriatal circuits in healthy G2019S LRRK2 carriers. Neurology. 2015;84(4):399-406.

33. Hughes AJ, Daniel SE, Kilford L, Lees AJ. Accuracy of clinical diagnosis of idiopathic Parkinson's disease: a clinic-pathological study of 100 cases. J Neurol Neurosurg Psychiatry. 1992;55:181-184.

34. Barone P, Antonini A, Colosimo C, et al. The PRIAMO study: a multicenter assessment of nonmotor symptoms and their impact on quality of life in Parkinson's disease. Mov Disord. 2009;24(11):1641-1649.

35. Erro R, Santangelo G, Picillo M, et al. Link between non-motor symptoms and cognitive dysfunctions in de novo, drug-naive PD patients J Neurol. 2012;259(9):1808-1813.

36. Picillo M, Amboni M, Erro R, et al. Gender differences in nonmotor symptoms in early, drug naïve Parkinson's disease. J Neurol. 2013;260(11):2849-2855.

37. Lewis SJG, Foltynie T, Blackwell AD, Robbins TW, Owen AM, Barker RA. Heterogeneity of Parkinson's disease in the early clinical stages using a data driven approach. J Neurol Neurosurg Psychiatry. 2005;76(3):343-348.

38. Marras C, Lang A. Parkinson's disease subtypes: lost in translation? J Neurol Neurosurg Psychiatry. 2013;84(4):409-415.

39. Erro R, Vitale C,Amboni M, et al. The heterogeneity of early Parkinson's disease: a cluster analysis on newly diagnosed untreated patients. PLoS One. 2013;8(8):e70244.

40. Nalls MA, McLean CY, Rick J, et al. Parkinson's Disease Biomarkers Program and Parkinson's Progression Marker Initiative investigators. Diagnosis of Parkinson's disease on the basis of clinical and genetic classification: a population-based modelling study. Lancet Neurol. 2015;14(10):1002-1009.

41. Köllensperger M, Geser F, Seppi K, et al. European MSA Study Group. Red flags for multiple system atrophy. Mov Disord. 2008; 23(8):1093-1099.

42. Todorović Z, Džoljić E, Novaković I, et al. Homocysteine serum levels and MTHFR C677T genotype in patients with Parkinson's disease, with and without levodopa therapy. J Neurol Sci. 2006;248(1-2): 56-61.

43. Levin J, Bötzel K, Giese A, Vogeser M, Lorenzl S. Elevated levels of methylmalonate and homocysteine in Parkinson's disease, progressive supranuclear palsy and amyotrophic lateral sclerosis. Dement Geriatr Cogn Disord. 2010;29:553-559.

44. Chen W, Cheng X, Zhang X, Zhang Q, Sun H. The expression features of serum Cystatin $\mathrm{C}$ and homocysteine of Parkinson's disease with mild cognitive dysfunction. Eur Rev Med Pharmacol Sci. 2015;19:2957-2963.
45. Scalzo P, Kümmer A, Bretas TL, Cardoso F, Teixeira AL. Serum levels of brain-derived neurotrophic factor correlate with motor impairment in Parkinson's disease. J Neurol. 2010;257(4):540-545.

46. Ziebell M, Khalid U, Klein AB, et al. Striatal dopamine transporter binding correlates with serum BDNF levels in patients with striatal dopaminergic neurodegeneration. Neurobiol Aging. 2012;33(2):428.e1-5.

47. Pellecchia MT, Pivonello R, Salvatore E, et al. Growth hormone response to arginine test distinguishes multiple system atrophy from Parkinson's disease and idiopathic late-onset cerebellar ataxia. Clin Endocrinol (Oxf). 2005;62(4):428-433.

48. Picillo M, Erro R, Santangelo G, et al. Insulin-like growth factor-1 and progression of motor symptoms in early, drug-naïve Parkinson's disease. J Neurol. 2013;260(7):1724-1730.

49. Pellecchia MT, Santangelo G, Picillo M, et al. Insulin-like growth factor-1 predicts cognitive functions at 2-year follow-up in early, drugnaïve Parkinson's disease. Eur J Neurol. 2014;21(5):802-807.

50. Liu Y, Zhang B-S. Serum 25-hydroxyvitamin D predicts severity in Parkinson's disease patients. Neurol Sci. 2014;35(1):67-71.

51. Palladino R, Moccia M, De Pascale T, et al. Season of birth and Parkinson's disease: possible relationship? Neurol Sci. 2015;36(8):1457-1462.

52. Gardener H, Gao X, Chen H, Schwarzschild MA, Spiegelman D, Ascherio A. Prenatal and early life factors and risk of Parkinson's disease. Mov Disord. 2010;25(11):1560-1567.

53. Mahlknecht P, Stemberger S, Sprenger F, et al. An antibody microarray analysis of serum cytokines in neurodegenerative Parkinsonian syndromes. Proteome Sci. 2012;10(1):71.

54. Moccia M, Picillo M, Erro R, et al. Increased bilirubin levels in de novo Parkinson's disease. Eur J Neurol. 2015;22(6):954-959.

55. Hatano T, Saiki S, Okuzumi A, Mohney R, Hattori N. Identification of novel biomarkers for Parkinson's disease by metabolomic technologies. J Neurol Neurosurg Psychiatry. Epub 2015 Mar 20.

56. Bharucha KJ, Friedman JK, Vincent AS, Ross ED. Lower serum ceruloplasmin levels correlate with younger age of onset in Parkinson's disease. J Neurol. 2008;255(12):1957-1962.

57. Jin L, Wang J, Zhao L, et al. Decreased serum ceruloplasmin levels characteristically aggravate nigral iron deposition in Parkinson's disease. Brain. 2011;134(1):50-58.

58. Shen C, Guo Y, Luo W, Lin C, Ding M. Serum urate and the risk of Parkinson's disease: results from a meta-analysis. Can J Neurol Sci. 2012;(88):73-79.

59. Shen L, Ji H-F. Low uric acid levels in patients with Parkinson's disease: evidence from meta-analysis. BMJ. 2013;3(11):e003620.

60. Schwarzschild MA, Marek K, Eberly S, et al. Serum urate and probability of dopaminergic deficit in early "Parkinson's disease". Mov Disord. 2011;26(10):1864-1868.

61. Moccia M, Pappatà S, Erro R, et al. Uric acid relates to dopamine transporter availability in Parkinson's disease. Acta Neurol Scand. 2015a;123(2):127-131.

62. Mariani S, Ventriglia M, Simonelli I, et al. Association between sex, systemic iron variation and probability of Parkinson's disease. Int $J$ Neurosci. 2015;126(4):354-360.

63. Fukushima T, Tan X, Luo Y, Kanda H. Relationship between blood levels of heavy metals and Parkinson's disease in China. Neuroepidemiology. 2010;34(1):18-24.

64. Simonsen A, Kuiperij B, Ali El-Agnaf O, et al. The utility of $\alpha$-synuclein as biofluid marker in neurodegenerative diseases: a systematic review of the literature. Biomark Med. Epub 2015 Aug 28.

65. Williams SM, Schulz P, Sierks MR. Oligomeric $\alpha$-synuclein and $\beta$-amyloid variants as potential biomarkers for Parkinson's and Alzheimer's diseases. Eur J Neurosci. Epub 2015 Aug 31.

66. Gupta V, Garg RK, Khattri S. Serological Analysis of Alpha-synuclein and NF- $\kappa \mathrm{B}$ in Parkinson's Disease Patients. J Clin Diagn Res. 2015;9(5):BC01-04.

67. Shi M, Liu C, Cook TJ et al. Plasma exosomal $\alpha$-synuclein is likely CNS-derived and increased in Parkinson's disease. Acta Neuropathol. 2014;128(5):639-650. 
68. Alves G, Pedersen KF, Bloem BR, et al. Cerebrospinal fluid amyloid- $\beta$ and phenotypic heterogeneity in de novo Parkinson's disease. J Neurol Neurosurg Psychiatry. 2013;84:537-543.

69. Stav AL, Aarsland D, Johansen KK, Hessen E, Auning E, Fladby T. Amyloid- $\beta$ and $\alpha$-synuclein cerebrospinal fluid biomarkers and cognition in early Parkinson's disease. Parkinsonism Relat Disord. 2015;21:758-764

70. Bibl M, Mollenhauer B, Esselmann H, et al. CSF amyloid- $\beta$-peptides in Alzheimer's disease, dementia with Lewy bodies and Parkinson's disease dementia. Brain. 2006;129:1177-1187.

71. van Dijk KD, Teunissen CE, Drukarch B, et al. Diagnostic cerebrospinal fluid biomarkers for Parkinson's disease: a pathogenetically based approach. Neurobiol Dis. 2010;39:229-241.

72. Jiménez-Jiménez FJ, Alonso-Navarro H, García-Martín E, Agúndez JAG. Cerebrospinal fluid biochemical studies in patients with Parkinson's disease: toward a potential search for biomarkers for this disease. Front Cell Neurosci. 2014;11:369.

73. Wennström M, Surova Y, Hall S, et al. Low CSF levels of both $\alpha$-synuclein and the $\alpha$-synuclein cleaving enzyme neurosin in patients with synucleinopathy. PLoS One. 2013;8:e53250.

74. Přikrylová Vranová H, Mareš J, Nevrlý M, et al. CSF markers of neurodegeneration in Parkinson's disease. J Neural Transm. 2010;117:1177-1181.

75. Waragai M, Wei J, Fujita M, et al. Increased level of DJ-1 in the cerebrospinal fluids of sporadic Parkinson's disease. Biochem Biophys Res Commun. 2006;345:967-972.

76. Herbert MK, Eeftens JM, Aerts MB, et al. CSF levels of DJ-1 and tau distinguish MSA patients from PD patients and controls. Parkinsonism Relat Disord. 2014;20:112-115

77. Kikuchi A, Takeda A, Onodera H, et al. Systemic increase of oxidative nucleic acid damage in Parkinson's disease and multiple system atrophy. Neurobiol Dis. 2002;9:244-248.

78. Buhmann C, Arlt S, Kontush A, et al. Plasma and CSF markers of oxidative stress are increased in Parkinson's disease and influenced by antiparkinsonian medication. Neurobiol Dis. 2004;15:160-170.

79. Öhman A, Forsgren L. NMR metabonomics of cerebrospinal fluid distinguishes between Parkinson's disease and controls. Neurosci Lett. 2015;594:36-39.

80. Trupp M, Jonsson P, Ohrfelt A, et al. Metabolite and peptide levels in plasma and CSF differentiating healthy controls from patients with newly diagnosed Parkinson's disease. J Parkinsons Dis. 2014;4(3):549-560.

81. Bajaj N, Hauser RA, Grachev ID. Clinical utility of dopamine transporter single photon emission CT (DaT-SPECT) with (123I) ioflupane in diagnosis of parkinsonian syndromes. J Neurol Neurosurg Psychiatry. 2013;84(11):1288-1295.

82. Niethammer M, Eidelberg D. Metabolic brain networks in translational neurology: concepts and applications. Ann Neurol. 2012;72(5): 635-647.

83. Chung EJ, Lee WY, Yoon WT, Kim BJ, Lee GH. Scintigraphy for differentiating Parkinson's disease with autonomic dysfunction from Parkinsonism-predominant multiple system atrophy. Mov Disord. 2009;24(11):1650-1655.

84. Reimão S, Pita Lobo P, Neutel D, et al. Substantia nigra neuromelanin magnetic resonance imaging in de novo Parkinson's disease patients. Eur J Neurol. 2015;22(3):540-546.

85. Zhang D, Liu X, Chen J, Liu B. Distinguishing patients with Parkinson's disease subtypes from normal controls based on functional network regional efficiencies. PLoS One. 2014;9(12):e115131.

86. Bunzeck N, Singh-Curry V, Eckart C, et al. Motor phenotype and magnetic resonance measures of basal ganglia iron levels in Parkinson's disease. Parkinsonism Relat Disord. 2013;19(12):1136-1142.

87. Moccia M, Pappatà S, Picillo M, et al. Dopamine transporter availability in motor subtypes of de novo drug-naïve Parkinson's disease. J Neurol. 2014;261(11):2112-2118.

88. Pellecchia MT, Picillo M, Santangelo G, et al. Cognitive performances and DAT imaging in early Parkinson's disease with mild cognitive impairment: a preliminary study. Acta Neurol Scand. 2015;131(5):275-281.
89. Erro R, Pappatà S, Amboni M, et al. Anxiety is associated with striatal dopamine transporter availability in newly diagnosed untreated Parkinson's disease patients. Parkinsonism Relat Disord. 2012;18(9):1034-1038.

90. Rajput AH, Sitte HH, Rajput A, Fenton ME, Pifl C, Hornykiewicz O. Globus pallidus dopamine and Parkinson motor subtypes: clinical and brain biochemical correlation. Neurology. 2008;70(16 Pt 2): 1403-1410.

91. Burn DJ, Rowan EN, Allan LM, Molloy S, O’Brien JT, McKeith IG. Motor subtype and cognitive decline in Parkinson's disease, Parkinson's disease with dementia, and dementia with Lewy bodies. $J$ Neurol Neurosurg Psychiatry. 2006;77(5):585-589.

92. Picillo M, Amboni M, Erro R, et al. Segmental progression of cardinal motor symptoms in Parkinson's disease: a pilot study suggesting a practical approach to rate disease course in the early stages. Parkinsonism Relat Disord. 2013;19(12):1143-1148.

93. Amboni M, Barone P, Picillo M, et al. A two-year follow-up study of executive dysfunctions in parkinsonian patients with freezing of gait at on-state. Mov Disord. 2010;25(6):800-802.

94. Amboni M, Cozzolino A, Longo K, Picillo M, Barone P. Freezing of gait and executive functions in patients with Parkinson's disease. Mov Disord. 2008;23(3):395-400.

95. Amboni M, Barone P, Iuppariello L, et al. Gait patterns in Parkinsonian patients with or without mild cognitive impairment. Mov Disord. 2012;27(12):1536-1543.

96. Picillo M, Dubbioso R, Iodice R, et al. Short-latency afferent inhibition in patients with Parkinson's disease and freezing of gait. J Neural Transm. 2015;122(11):1533-1540.

97. Erro R, Picillo M, Vitale C, et al. Non-motor symptoms in early Parkinson's disease: a 2-year follow-up study on previously untreated patients. J Neurol Neurosurg Psychiatry. 2013;84(1):14-17.

98. Picillo M, Erro R, Amboni M et al. Gender differences in nonmotor symptoms in early Parkinson's disease: a 2-years follow-up study on previously untreated patients. Parkinsonism Relat Disord. 2014;20(8):850-854.

99. Santangelo G, Vitale C, Picillo M, et al. Mild Cognitive Impairment in newly diagnosed Parkinson's disease: A longitudinal prospective study. Parkinsonism Relat Disord. 2015;21(10):1219-1226.

100. Picillo M, Barone P, Pellecchia MT, et al. Evolution of mild cognitive impairment in Parkinson disease. Neurology. 2014;82(15):1384.

101. Santangelo G, Vitale C, Trojano L, et al. Relationship between apathy and cognitive dysfunctions in de novo untreated Parkinson's disease: a prospective longitudinal study. Eur J Neurol. 2015;22(2):253-260.

102. Erro R, Santangelo G, Barone P, et al. Do subjective memory complaints herald the onset of mild cognitive impairment in Parkinson disease? J Geriatr Psychiatry Neurol. 2014;27(4):276-281.

103. Manganelli F, Vitale C, Santangelo G, et al. Functional involvement of central cholinergic circuits and visual hallucinations in Parkinson's disease. Brain. 2009;132(Pt 9):2350-2355.

104. Moccia M, Mollenhauer B, Erro R, Picillo M, Palladino R, Barone P. Non-Motor Correlates of Smoking Habits in de Novo Parkinson's Disease. J Parkinsons Dis. 2015;5(4):913-924.

105. Kotagal V, Albin RL, Müller ML, Koeppe RA, Frey KA, Bohnen NI. Modifiable cardiovascular risk factors and axial motor impairments in Parkinson disease. Neurology. 2014;82:1514-1520.

106. Costa A, Peppe A, Carlesimo GA et al. Brain-derived neurotrophic factor serum levels correlate with cognitive performance in Parkinson's disease patients with mild cognitive impairment. Front Behav Neurosci. 2015;9:253.

107. Chen-Plotkin AS, Hu WT, Siderowf A et al. Plasma EGF levels predict cognitive decline in Parkinson's disease. Ann Neurol. 2011;69(4): 655-663

108. Pellecchia MT, Santangelo G, Picillo M, et al. Serum epidermal growth factor predicts cognitive functions in early, drug-naive Parkinson's disease patients. J Neurol. 2013;260(2):438-444.

109. Meamar R, Shaabani P, Tabibian SR, Aghaye Ghazvini MR, Feizi A. The effects of uric acid, serum vitamin D3, and their interaction on Parkinson's disease severity. Parkinsons Dis. Epub 2015 Feb 24. 
110. Dufek M, Rektorova I, Thon V, Lokaj J, Rektor I. Interleukin-6 May Contribute to Mortality in Parkinson's Disease Patients: A 4-Year Prospective Study. Parkinsons Dis. Epub 2015 Aug 16.

111. Hofmann KW, Schuh AFS, Saute J, et al. Interleukin-6 serum levels in patients with Parkinson's disease. Neurochem Res. 2009;34(8):1401-1404.

112. Tang P, Chong L, Li X, et al. Correlation between serum RANTES levels and the severity of Parkinson's disease. Oxid Med Cell Longev. 2014;2014:208408.

113. Jiménez-Jiménez F, Molina J, de Bustos F, et al. Serum levels of coenzyme Q10 in patients with Parkinson's disease. J Neural Transm. 2000;107:177-181.

114. Ascherio A, LeWitt $\mathrm{P}, \mathrm{Xu} \mathrm{K}$, et al. Urate predicts rate of clinical decline in Parkinsons disease. Arch Neurol. 2009;66(12):1460-1468.

115. Zhang H, Guo J, He D, et al. Lower serum UA levels in Parkinson's disease patients in the Chinese population. Neurosci Lett. 2012;514(2):152-155.

116. Jesús S, Pérez I, Cáceres-Redondo MT, et al. Low serum uric acid concentration in Parkinson's disease in southern Spain. Eur J Neurol 2013;20(1):208-210.

117. Pan M, Gao H, Long L et al. Serum uric acid in patients with Parkinson's disease and vascular parkinsonism: a cross-sectional study. Neuroimmunomodulation. 2013;20(1):19-28.

118. Lolekha P, Wongwan P, Kulkantrakorn K. Association between serum uric acid and motor subtypes of Parkinson's disease. J Clin Neurosci. 2015;22(8):1264-1267.

119. Fukae J, Ishikawa K, Hatano T, et al. Serum uric acid concentration is linked to wearing-off fluctuation in Japanese Parkinson's disease patients. J Parkinsons Dis. 2014;4(3):499-505.

120. Moccia M, Picillo M, Erro R, et al. Presence and progression of nonmotor symptoms in relation to uric acid in de novo Parkinson's disease. Eur J Neurol. 2015;22(1):93-98.

121. Moccia M, Picillo M, Erro R, et al. Is serum uric acid related to non-motor symptoms in de-novo Parkinson's disease patients? Parkinsonism Relat Disord. 2014;20(7):772-775.

122. Annanmaki T, Pessala-Driver A, Hokkanen L, Murros K. Uric acid associates with cognition in Parkinson's disease. Parkinsonism Relat Disord. 2008;14(7):576-578.

123. Annanmaki T, Pohja M, Parviainen T, Hakkinen P, Murros K. Uric acid and cognition in Parkinson's disease: a follow-up study. Parkinsonism Relat Disord. 2011;17(5):333-337.

124. Schwarzschild MA, Ascherio A, Beal MF, et al. Inosine to increase serum and cerebrospinal fluid urate in Parkinson disease: a randomized clinical trial. JAMA Neurol. 2014;71(2):141-150.

125. Yu SY, Sun L, Liu Z, et al. Sleep disorders in Parkinson's disease: clinical features, iron metabolism and related mechanism. PLoS One. 2013;8(12):e82924.

126. Hu Y, Yu SY, Zuo L-J, et al. Investigation on Abnormal Iron Metabolism and Related Inflammation in Parkinson Disease Patients with Probable RBD. PLoS One. 2015;10(10):e0138997.
127. Fukushima T, Tan X, Luo Y, et al. Heavy metals in blood and urine and its relation to depressive symptoms in Parkinson's disease patients. Fukushima J Med Sci. 2013;59(2):76-80.

128. van Dijk KD, Bidinosti M, Weiss A, Raijmakers P, Berendse HW, van de Berg WDJ. Reduced $\alpha$-synuclein levels in cerebrospinal fluid in Parkinson's disease are unrelated to clinical and imaging measures of disease severity. Eur J Neurol. 2014;21(3):388-394.

129. Hall S, Surova Y, Öhrfelt A, Zetterberg H, Lindqvist D, Hansson O. CSF biomarkers and clinical progression of Parkinson disease. Neurology. 2015;84:57-63.

130. Liu C, Cholerton B, Shi M, et al. CSF tau and tau/A $\beta 42$ predict cognitive decline in Parkinson's disease. Parkinsonism Relat. Disord. 2015;21:271-276.

131. Kang JH, Chen-Plotkin AS. SiderowfA. Association of cerebrospinal fluid $\beta$-amyoid 1-42, T-tau, P-tau181, and $\alpha$-synuclein levels with clinical features of drug-naive patients with early Parkinson disease. JAMA Neurol. 2013;70(10):1277-1287.

132. OldeDubbelink KT, Schoonheim MM, Deijen JB, Twisk JW, Barkhof F, Berendse HW. Functional connectivity and cognitive decline over 3 years in Parkinson disease. Neurology. 2014;83(22):2046-2053.

133. Amboni M, Tessitore A, Esposito F, et al. Resting-state functional connectivity associated with mild cognitive impairment in Parkinson's disease. J Neurol. 2015;262(2):425-434.

134. Tessitore A, Amboni M, Esposito F, et al. Resting-state brain connectivity in patients with Parkinson's disease and freezing of gait. Parkinsonism Relat Disord. 2012;18(6):781-787.

135. Jia X, Liang P, Li Y, Shi L, Wang D, Li K. Longitudinal Study of Gray Matter Changes in Parkinson Disease. AJNR Am J Neuroradiol. 2015;36(12):2219-2226.

136. Ibarretxe-Bilbao N, Junque C, Segura B, et al. Progression of cortical thinning in early Parkinson's disease. J Neurol Neurosurg Psychiatry. 2013;84(8):875-881.

137. Zarei M, Ibarretxe-Bilbao N, Compta $\mathrm{Y}$ et al. Cortical thinning is associated with disease stages and dementia in Parkinson's disease. Mov Disord. 2012;27(14):1746-1753.

138. Ulla M, Bonny JM, Ouchchane L, Rieu I, Claise B, Durif F. Is R2* a new MRI biomarker for the progression of Parkinson's disease? A longitudinal follow-up. PLoS One. 2013;8(3):e57904.

139. McKeown MJ, Peavy GM. Biomarkers in Parkinson disease: it's time to combine. Neurology. 2015;84(24):2392-2393.

140. Campbell MC, Koller JM, Snyder AZ, Buddhala C, Kotzbauer PT, Perlmutter JS. CSF proteins and resting-state functional connectivity in Parkinson disease. Neurology. 2015;84(24):2413-2421.

141. Rosenthal LS, Drake D, Alcalay RN, et al. PDBP consortium. The NINDS Parkinson's disease biomarkers program. Mov Disord. Epub 2015 Oct 7.
Journal of Parkinsonism \& Restless Legs Syndrome

\section{Publish your work in this journal}

Journal of Parkinsonism and Restless Legs Syndrome is an online, open access, peer-reviewed journal. The journal publishes review articles, historical reviews, original research articles, case reports, letters to the editor, clinical teaching cases, neuroradiology highlights, neuropathology highlights, neuropsychiatry highlights, autobiographies, conference

\section{Dovepress}

proceedings, abstracts and book reviews. The manuscript management system is completely online and includes a very quick and fair peerreview system, which is all easy to use. Visit http://www.dovepress.com/ testimonials.php to read real quotes from published authors. 